\title{
Philosophiques
}

\section{Gramsci et la question de l’idéologie}

\section{Yvan Cloutier}

Volume 10, numéro 2, octobre 1983

Le marxisme cent ans après Marx

URI : https://id.erudit.org/iderudit/203228ar

DOI : https://doi.org/10.7202/203228ar

Aller au sommaire du numéro

Éditeur(s)

Société de philosophie du Québec

ISSN

0316-2923 (imprimé)

1492-1391 (numérique)

Découvrir la revue

Citer cet article

Cloutier, Y. (1983). Gramsci et la question de l’idéologie. Philosophiques, 10(2), 243-253. https://doi.org/10.7202/203228ar d'utilisation que vous pouvez consulter en ligne.

https://apropos.erudit.org/fr/usagers/politique-dutilisation/ 


\title{
GRAMSCI ET LA QUESTION DE L'IDÉOLOGIE
}

\author{
par Yvan Cloutier
}

Le format table ronde ${ }^{1}$ vise davantage la problématisation que l'exposition scientifique, d'où la brièveté et le caractère " général " des interventions. L'entreprise se complique lorsqu'il s'agit d'y aborder l'œuvre d'Antonio Gramsci ; cette œuvre est «asystématique ${ }^{2}$, «instrumentalisée ${ }^{3}$ politiquement dans l'histoire de son édition ${ }^{4}$ et par la diversité des lectures ${ }^{5}$, et une œuvre encore sous-analysée d'un point de vue scientifique. Toute entreprise d'élucidation d'un concept devient complexe : il faut isoler ce concept, c'est-à-dire " abstraire " (ici construire le concept d'idéologie en faisant abstraction du concept d'hégémonie) et toute intervention sur un concept risque d'entraîner une relecture des autres concepts connexes.

1. Ce texte a fait l'objet d'une communication dans l'atelier « Les formes philosophiques du marxisme et la théorie de l'idéologie " lors du Congrès de l'ACFAS mai 1983.

2. Gramsci signale à quelques reprises le caractère provisoire et approximatif des notes des Cabiers de prison ; elles " ont été écrites au courant de la plume, en vue de former un rapide aide-mémoire. Elles sont toutes à revoir et à contrôler minutieusement . . . ". Antonio Gramsci, Quaderni del carcere, Torino, Finaudi Editore, Edizione critica dell'Istituto Gramsci, a cura di V. Gerratana, 1975, volume II, 1978, p. 1365 ; traduction : Cahiers de prison, cabiers 10, 11, 12, 13, Paris, Gallimard (coll. Bibliothèque de philosophie), 1978, p. 165 ; voir aussi les Quaderni p. 438 et p. 935. Cette asystématicité donne lieu à des interprétations parfois inconciliables de thèmes comme ceux de la société civile et de l'hégémonie.

3. Dans l'acception italienne du mot "strumentalizarre" : se servir de qqch. ou de qqn. pour atteindre ses propres fins ; nous empruntons l'expression à Giorgio Bonomi, Partito e rivoluzıone in Gramsci, Milano, Feltrinelli Editore, seconda edizione 1976 (c 1973), pp. 910.

4. L'édition intégrale et chronologique des Quadernı date de 1975. L'éditeur Finaudi avait publié de 1948 à 1951 une édition incomplète des notes organisées en six tomes autour de thèmes choisis par $P$. Togliatti qui était à la fois responsable de l'édition, exégète et secrétaire du P.C.I..

5. Voir : Gian Carlo Jocteau, Leggere Gramsci, Guida alle interpretazion, Milano, Feltrinelli Economica, terza edizione 1977 (c 1975), 180 pp. ; Mario Biondi, Guida Bibliographica a Gramsci, Cesena, Lıbreria Adamo Bittini [s.d. (1977 ?)], 49 p. ; Jacques Texier, Notes sur Gramscu (à propos de quelques articles), Paris, Les Cahiers du Centre d'études et de recherches marxistes, $1974,37 \mathrm{p}$. 


\section{LA QUESTION DE PERTINENCE}

Quelle est la pertinence d'une lecture de Gramsci pour les fins de notre rencontre qui porte sur « Les formes philosophiques du marxisme et la théorie de l'idéologie "?

L'œuvre gramscienne s'inscrit en rupture dans le marxisme non seulement en ce qui a trait aux thématisations philosophiques (critique du matérialisme dialectique, la gnoséologie, la langue, etc.) mais en particulier en ce qui concerne le statut même du philosophique et de l'idéologique.

L'originalité de Gramsci réside dans sa formulation d'une définition neutre et extensive de l'idéologique - le champ idéologique comprend les représentations implicites et explicites, et ces représentations ne sont pas par définition déformantes, occultantes ni réactionnaires. $\grave{A}$ un niveau d'analyse plus spécifique, Gramsci pose des déterminations restreintes (articulé/inarticulé, implicite/explicite, contenus dégradés/contenus originaux, passif/actif, progressiste/réactionnaire, etc. ${ }^{6}$ ) et étudie les rapports entre les niveaux (ou degrés quantitatifs) du champ idéologique ${ }^{7}$. À l'intérieur de ce champ idéologique, le niveau philosophique remplit une fonction privilégiée ; comme le processus idéologique vise à la diffusion d'une conception du monde à laquelle est liée une pratique, le travail du philosophe sera un travail d'éducation du sens commun afin de le rendre plus cohérent, plus actif et surtout plus organique (l'organicité étant la condition de possibilité de la progressivité d'une idéologie). En somme, le travail du philosophe doit se situer dans le champ idéologique et non en marge; de plus l'enjeu de l'hégémonie philosophique est un enjeu politique ${ }^{8}$, d'où une valorisation du travail philosophique chez Gramsci.

6. Voir : Alberto M. Cirese, "Concezioni del mondo, filosofia spontanea, folclore ", in AA. VV., Gramsci e la cultura contemporanea, Roma, Editori Riuniti - Istituto Gramsci, 1969, vol. 2, pp. 299-328 ; traduction : " Conceptions du monde, philosophie spontanée, folklore ", Dialectiques, spécial Gramsci, n 3-4, 1974, pp. 83-91.

7. Ces niveaux sont la philosophie, la religion, le " bon sens ", le sens commun et le folklore. Sur la différence "quantitative ", voir : Q. 10, I, [10], p. 1231 (traduction : C. p. 37) et Q. 10 II, [2], p. 1241 (trad. : C. p. 47) ; nous désignons l'édition italienne des Quaderni par $Q$. et la traduction Gallimard par $C$.

8. Pour une analyse des rapports entre devenir culturel des masses et leur devenir politique, voir : Giorgio Nardone, Il pensiero di Gramsci, Bari, De Donato, 1971, en particulier la section intitulée " Il divenire culturale della massa ", pp. 185-223. 
La reconstruction critique du concept d'idéologie part du constat suivant : "le sens défavorable (deteriore) du mot est devenu extensif et ce fait a modifié et dénaturé l'analyse théorique du concept d'idéologie. " $"$. Selon Gramsci, cette acception péjorative est dominante et se caractérise par une conception épiphénoméniste des idéologies ; il mènera la lutte sur deux fronts : B. Croce et $\mathrm{N}$. Boukharine.

Croce réduit l'idéologie à un simple discours-outil politique, c'est le lieu du " manipulatoire "; la déformation/occultation y est intentionnelle, c'est-à-dire qu'il faut distinguer entre, d'une part, la victime de l'idéologie qui appartient à la catégorie intellectuelle des simples et à la catégorie politique des dominés et, d'autre part, le dominant, cultivé ou philosophe, qui pour cette raison est hors de l'idéologie ${ }^{10}$.

Quant à Boukharine, il définit l'idéologie comme système de représentations déformantes; la déformation étant essentielle, l'idéologie devient le lieu de l'« illusoire » dans lequel tant le simple que le cultivé, le dominant que le dominé subissent l'effet d'occultation; la question de la scientificité dépend alors de la reconnaissance/méconnaissance de la base matérielle. La difficulté d'une telle dichotomie tient à la justification de la prétention à la scientificité par laquelle le discours de l'autre se voit rejeté dans l'idéologie ${ }^{11}$.

\section{UNE CONCEPTION NEUTRE ET EXTENSIVE}

Ces conceptions épiphénoménistes de l'idéologie « dénature[nt] l'analyse théorique du concept d'idéologie " parce qu'en restreignant «a priori » le champ idéologique, elles limitent la

9. Voir : Q. 7, [19], p. 868.

10. " ce qui est important pour Croce, c'est que les intellectuels ne s'abaissent pas au niveau de la masse mais qu' ils comprennent que l'idéologie est une chose, un instrument pratique pour gouverner et que la philosophie et la religion en sont une autre, qu'il ne faut pas prostituer dans la conscience des prêtres. Les intellectuels doivent être des gouvernants et non des gouvernés, des constructeurs d'idéologies pour gouverner les autres et non des charlatans qui se laissent mordre et empoisonner par leurs propres vipères. ». Q. 10, I, [1], p. 1212 , C. p. 20.

11. Fernand Dumont soulève cette connotation polémique du concept d'idéologie ; "Officiellement [écrit-il] l'idéologie est la pensée de l'autre. ", Les idéologies, Paris, Presses Universitaires de France, 1974, p. 6. 
fonction des idéologies et par conséquent elles escamotent l'épineuse question de la place de la lutte idéologique dans le processus révolutionnaire.

Gramsci préfere une définition extensive et génériquement neutre ${ }^{12}$ du concept : l'idéologie est une conception du monde accompagnée d'une norme de conduite conforme. Dans un deuxième temps une idéologie sera analysée plus spécifiquement à plusieurs points de vue : celui du processus de systématisation (cohérent vs incohérent, implicite vs explicite, etc.), celui des modalités de contraste (opposition, contradiction) par rapport à d'autres conceptions du monde (conception tenace, effective, expansive, etc.), et celui du caractère progressif ou réactionnaire ${ }^{13}$. Ce n'est qu'au terme de l'analyse qu'une idéologie peut être qualifiée de péjorative ou positive, de progressive ou réactionnaire ; une conception du monde même si elle est implicite et inarticulée peut être tenace, expansive et progressive ${ }^{14}$ à la condition, comme nous le verrons plus loin, qu'elle soit organique et qu'elle soit élaborée, c'est-à-dire qu'elle soit travaillée par le philosophe (qui, pour Gramsci, est une politique ${ }^{15}$ ).

Revenons à la définition gramscienne de l'idéologie; Gramsci a élaboré son concept d'idéologie à partir du concept crocien de religion «entendu non dans le sens confessionnel, mais dans le sens d'une unité de foi entre une conception du monde et une norme de conduite qui lui soit conforme . . . " ${ }^{16}$. Dès lors il n'y aura pas de différence qualitative mais quantitative entre ces degrés du champ idéologique que sont la philosophie,

12. Lorsque Gramsci veut désigner une idéologie dans l'acception restrictive et péjorative il emploie les qualificatifs et les propositions subordonnées appropriés. Exemples : « une idéologie dans le sens péjoratif (deteriore) " Q. 10, II, [41], XIV, p. 1325, C. p. 124 ; " le carcan des idéologies (au sens péjoratif, de fanatisme aveugle) »Q. 10, II, [24], p. 1263 , C. p. 68 ; " une idéologie dans le sens le plus mauvais (deteriore), c'est-à-dire un système dogmatique de vérités absolues et éternelles . . . \ Q. 11, [62], p. 1489, C. p. 285 . Voir aussi : Q. 11, [62], p. 1487, C. p. 283 ; Q. 10, I, [10], p. 1229, C. p. 35 ; Q. 14 [68], p. 1729 et Q. 16, [9], p. 1864.

13. Voir : A.M. Cirese, op. cit..

14. D'où la possibilité d'une idéologie « prolétarienne " même si elle est une idéologie dominée. Voir : Bernard Labrousse, De l'idéologie dominée, Montréal, Les Éditions Nouvelle Optique, 1978 ; sur Gramsci et les idéologies dominées, pp. 85-89.

15. Voir : Q. 10, II, [2], p. 1241, C. p. 47.

16. «. . . mais pourquoi appeler cette unité de foi " religion " et ne pas l'appeler « idéologie ", ou carrément " politique "? "Q. 11, [12], note IV, p. 1378, C., p. 178. Voir aussi : Q. 10, II, [41], 1, p. 1293, C., p. 95 ; Q. 10, I, [5], p. 1217, C. pp. 24-25 ; Q. 10, II, [14], p. 1251, p. 57. 
la religion, le «bon sens », le sens commun et le folklore si on ne les « considère plus comme une activité théorique (création d'une nouvelle pensée) mais comme un stimulant à l'actioǹ (activité éthico-politique concrète, création d'une nouvelle histoire). ${ }^{17}$. Une telle extension du concept d'idéologie rend possible l'analyse du champ idéologique en termes d'un " continuum » entre divers moments ${ }^{18}$ d'où la possibilité du travail d'élaboration idéologique.

La réflexion des Cabiers de prison porte sur les problèmes du passage au socialisme en Occident et plus particulièrement sur l'échec italien, d'où l'accent sur la question des superstructures. L'intérêt du travail gramscien sur l'idéologie réside dans ses nombreuses analyses d'idéologies particulières tels l'étude des programmes des divers partis politiques autour du Risorgimento, l'influence de l'Église, la pensée de Machiavel, le rôle des Jacobins dans la Révolution française, etc.. Malheureusement, les Cabiers qui contiennent la plupart de ces analyses ne sont pas encore accessibles au lecteur français. Gramsci met en place des concepts qu'il expérimente et qu'il réajuste sans avoir eu l'occasion de reprendre systématiquement toute cette question des idéologies. Nous proposons un essai de systématisation autour de la définition suivante dont nous expliciterons par la suite certains éléments : une idéologie est une conception du monde (implicite ou explicite) à laquelle est liée une norme de conduite; cette conception du monde a une diffusivité (aspect de masse) et a ou a eu ou aura un efficace (aspect passionnel) en ce qu'elle est un stimulant à l'action des individus dans une classe sociale donnée.

\section{L'IMPLICITE ET L'EXPLICITE}

D'après les définitions courantes, ${ }^{19}$ l'idéologie ne concernerait que les conceptions du monde explicites, voire articulées de manière à pouvoir fonctionner comme rationalisation ; que

17. Q. 10, I, [5], p. 1217 , C. pp. 24-25; aussi Q. 11, [12], p. 1380, C. p. 180 : « Mais c'est en ce point que se pose le problème fondamental de toute conception du monde, de toute philosophie qui est devenue un mouvement culturel, une "religion", une "foi ", c'est-à-dire qui a produit une activité pratique et une volonté . . ."

18. " [Peut-être est-il utile " pratiquement " de distinguer la philosophie du sens commun, pour mieux indiquer le passage de l'un de ces moments à l'autre. » Q. 11, [12], note IV, p. 1382 , C. p. 182.

19. Une idéologie désigne, tant chez R. Aron, Althusser que F. Dumont, un système ou un complexe de représentations doté d'un minimum de cohérence. 
fait-on des représentations implicites véhiculées dans les pratiques individuelles et collectives? Pour Gramsci, l'idéologie est une " conception du monde qui se manifeste implicitement dans l'art, dans le droit, dans l'activité économique, dans toutes les manifestations de la vie individuelle et collective . . . ${ }^{20}$. Considérons brièvement en quoi il y a une conception du monde implicite dans la langue, l'art et la science.

Contre Manzoni et sa conception instrumentale de la langue, Gramsci affirme une identité entre langue et culture ${ }^{21}$; « toute langue, écrit-il, est une conception du monde intégrale, et non seulment un vêtement (vestito) qui s'ajuste indifféremment de la forme et de tout contenu. ". Il y a corrélation entre une langue et la complexité d'une conception du monde ${ }^{22}$ d'où par exemple les limites du dialecte par rapport à une langue nationale dans l'accès à la culture mondiale et dans l'appréhension de la situation actuelle. Les jacobins ont compris l'importance du processus d'unification linguistique comme condition de la formation d'une volonté collective nationale populaire. D'où l'importance de la question de la langue dans la lutte idéologique :

la langue doit être traitée comme une conception du monde, comme l'expression d'une conception du monde ; le perfectionnement technique de l'expression soit quantitative (acquisition de nouveaux moyens d'expression), soit qualitative (acquisition de nuances dans la signification et d'un ordre syntaxique et stylistique plus complexe) signifie un élargissement et un approfondissement de la conception du monde et de son histoire . . . ${ }^{23}$

La critique littéraire a occupé une place importante dans le travail journalistique de Gramsci et dans ses Cabiers de Prison; il s'employait à expliciter la conception du monde implicite dans les œuvres littéraires. Notamment, il montra comment la conception du monde implicite dans l'œuvre pirandellienne ${ }^{24}$ est subjectiviste et par là s'oppose aux catholiques quoique cette conception du monde ne soit pas «cohérente » et qu'on ne

20. Q. 11, [12], note IV, p. 1380 , C. p. 180.

21. Voir : Franco Lo Piparo, Lingua, Intellettuali, Egemonia in Gramsci, Bari, Laterza, 1979, $292 \mathrm{p}$.

22. Q. 11, [12], note III, p. 1377, C. p. 177

23. Q. 5, [131], p. 664.

24. Sur Pirandello: Q. 6, 26, p. 705 ; Q. 9, 134, pp. 1195-1197 ; Q. 14, [15], pp. 16701674. 
puisse pas en tirer une philosophie. Pourquoi Gramsci s'intéressa-t-il tant à la littérature populaire sinon parce que le succès d'un livre " indique » (et est souvent le seul indicateur existant) quelle est la " philosophie de l'époque », c.-à-d. quelle masse de sentiments et de conceptions du monde prédomine dans la majorité $"$ silencieuse $" ~ " 25$.

La science elle-même est « une surperstructure, une idéologie "; " nonobstant tous les efforts des savants, [elle] ne se présente jamais comme une notion objective : elle apparaît toujours revêtue d'une idéologie, et concrètement ce qui est science c'est l'union du fait objectif avec une hypothèse qui dépassent le pur fait objectif. ${ }^{26}$. La science relève de la culture (d'une conception du monde) non seulement par l'historicité des instruments matériels et logiques de discrimination et d'établissement de la certitude ${ }^{27}$, mais surtout par les théories ou hypothèses qu'elle formule. ${ }^{28}$

Cet élargissement du champ idéologique est d'une utilité pédagogico-politique considérable. $S$ ' « il ne s'agit pas d'introduire ex novo une science dans la vie individuelle de " tout le monde ", mais de renouveler et de rendre "critique » une activité déjà existante . . . . ${ }^{29}$, seule la connaissance du sens commun permettra de vaincre l'inertie des « croyances populaires" et d'utiliser politiquement certains éléments comme cet « esprit de scission" (ou «instinct de classe ») caractéristique du folklore $^{30}$. La catégorie d'implicite permet aussi l'analyse des phénomènes de « dissonance " entre la conception du monde explicite (rationalisation) et la conception du monde implicite (dans la pratique des individus et des collectivités ${ }^{31}$ ), en d'autres termes entre la conception déclarée et la conception réelle ou

25. Q. 5, [54], p. 587.

26. Q. 11, [38], p. $1457-1458$, C. pp. 254-255

27. Q. 11, [37], pp. 1455-1456, C. pp. 252-253.

28. Gramsci prend comme exemple la théorie atomique, Q. 11, [30], pp. 1444-1445, C. p. 242.

29. Q. 11, [12], note IV, p. 1383, C. pp. 182-183.

30. L'activité formative de l'État doit inclure l'enseignement du folklore pour le dépasser ; Q. 27, [1], p. 2314.

31. "Tout État a " deux " philosophies celle qui s'énonce dans des formules et est un simple art de gouverner, et celle qui s'affirme avec l'action et est la philosophie réelle, c'est-àdire l'histoire. Le problème est de voir dans quelle mesure ces deux philosophies coïncident ou divergent. ", Q. 1, [87], p. 88. 
effective $^{32}$. Le chercheur sera face à des conceptions du monde composées d'éléments disparates et souvent contradictoires qui empêchent l'individu et le groupe de comprendre leur situation et de la transformer :

Quand la conception que l'on a du monde n'est ni critique ni cohérente, mais désagrégée et occasionnelle, on appartient simultanément à une multiplicité d'hommes-masses, la personnalité est composée de façon bizarre : on y trouve des éléments de l'homme des cavernes et des principes de la science la plus moderne et la plus avancée, on y trouve les préjugés de toutes les phases historiques passées avec l'étroitesse de leur localisation, pêle-mêle avec les intuitions d'une philosophie à venir (. . .) Comment est-il possible de penser le présent, et un présent bien déterminé, avec une pensée élaborée pour les problèmes d'un passé souvent bien lointain et bien dépassé ?33 $^{33}$

Philosopher, ce sera dès lors rendre critique sa conception du monde et par là être à même pour un individu et un groupe de comprendre ses tâches et être mieux outillé pour y répondre.

\section{LA DIFFUSIVITÉ $^{34}$ (L'ASPECT DE MASSE)}

Toute idéologie est une conception du monde, mais toute conception du monde n'est pas une idéologie ; il manquera aux "élucubrations individuelles ${ }^{35}$ et arbitraires du philosophe « $\mathrm{X}$ » le caractère de diffusivité (ou d'expansivité) essentiel à toute idéologie. Il y a des philosophies individuelles mais pas d'idéologie individuelle ${ }^{36}$; cependant notre philosophe " $\mathrm{X}$ » participera à une conception du monde implicite (celle de sa pratique) et sa conception explicite sera tributaire de d'autres idéologies.

Toute philosophie, et en particulier la philosophie de la praxis, a un " caractère tendanciel de philosophie de masse ${ }^{37}$,

\footnotetext{
32. Q. 11, [12], note IV, pp. 1378-1379, C. pp. 178-179.

33. Q. 11, [12], notes I et II, pp. 1378-1379 ; C. pp. 178-179.

34. Ce terme emprunté à l'italien " diffusività " est employé dans la traduction Gallimard, p. 182.

35. L'expression est dans Gramsci Q. 15, [25], p. 1782.

36. La philosophie crocienne de la liberté ne devint pas une idéologie, son "absence d' « expansivité " dans les grandes masses, témoigne du caractère restreint, immédiatement pratique de la philosophie de la liberté. ", Q. 10, I, [10], p. 1232.

37. Q. 11, [13], p. 1397, C. p. 196
} 
elle aspire à devenir un nouveau sens commun. C'est par sa diffusion qu'elle devient une idéologie et qu'elle a un efficace :

... ce sont les idéologies qui seront alors la « vraie " philosophie puisqu'elles s'avèreront être ces « vulgarisations " philosophiques qui poussent les masses à l'action concrète . . . Elles seront l'aspect de masse de chaque conception philosophique qui, chez le « philosophe » prend des caractères particuliers d'origine littéraire et antihistorique. ${ }^{38}$

\section{L'EFFICACE $^{39}$ (L'ASPECT PASSIONNEL)}

Ce qui importe n'est pas l'opinion de Pierre, Paul ou Jacques, mais cet ensemble d'opinions qui sont devenues collectives, devenues un élément et une force sociale ; ce sont elles qu'il convient de réfuter ... sans s'imaginer pour autant que l'on a déjà « détruit » ainsi l'élément et la force sociale correspondants (ce qui serait du rationalisme des Lumières à l'état pur). ${ }^{40}$

Une conception du monde devient une idéologie lorsqu'elle devient « un stimulant à l'action (activité éthico-politique concrète, création d'une nouvelle histoire) ", c'est-à-dire lorsque le rapport théorie/pratique atteint cette unité caractéristique de la « foi ». Cette unité confere aux croyances populaires ce caractère de " compacitié granitique ", c'est-à-dire " la même énergie que les forces matérielles $» .{ }^{41}$

La «prédominance idéologique » est une prédominance " émotive $"{ }^{42}$; l'idéologie a un efficace, elle fait agir parce qu'elle " élabore " des sentiments et des passions. Dans les "petites notes sur la politique de Machiavel ${ }^{43}$, Gramsci décrit les procédés discursifs utilisés par Machiavel et leurs effets; par le recours à la forme dramatique du mythe, Machiavel combine savoir et sentir, les concepts y sont personnifiés (le « condottiere » est une représentation plastique et anthropomorphique de la volonté collective). "Il semble, écrit Gramsci, que tout

38. Q. 10, II, [2], p. 1242, C. pp. 47-48.

39. Gramsci emploie l'expression à plusieurs reprises, entre autres : " della reale efficienza dei nessi ideologici » (Q. 5, 126, p. 655); dans Q. 6, [85], p. 759, il note que la doctrine politique de Dante " non ha avuto nessuna efficacia e fecondità « storico-culturale " ".

40. Q. 11, [15], pp. 1403-1404, C. p. 204.

41. Q. 11, [62], p. 1487, C. 282-283.

42. Dans Q. 7, [103], p. 929 : « . . il predominio ideologico (o emotivo) . . " .

43. Q. 13 . 
le travail " logique " ne soit qu'une réflexion du peuple sur luimême, un raisonnement intérieur, qui s'opère dans la conscience populaire et qui trouve sa conclusion dans un cri passionné, immédiat. De raisonnement sur elle-même, la passion redevient " affect ", fièvre, fanatisme d'action. " ${ }^{44}$. L'instrumentalisation gramscienne de Machiavel vise à faire "sentir » le savoir que Gramsci tire du jacobinisme ; cette «traduction » italienne du jacobinisme est pédagogico-politique, il s'agit pour Gramsci de mettre en mouvement les énergies nécessaires pour la création d'une volonté collective nationale populaire sous la direction du Prince moderne, le Parti.

\section{CONCLUSIONS}

Nous avons éclairé la définition gramscienne de l'idéologie afin d'en révéler l'originalité (à l'intérieur du marxisme) et le caractère opérationnel. En guise de conclusion nous formulons quelques brèves remarques sur le concept d'organicité et sur le travail du philosophe.

Qu'est-ce qui fait qu'une philosophie soit devenue ou devienne un idéologie alors que la plupart des philosophies des "professionnels" de la philosophie ne dépassent pas le stade des "élucubrations individuelles" et arbitraires?

Lorsqu'une philosophie devient idéologie, elle devient "réelle ${ }^{45}$, «ultra-réaliste ${ }^{46}$, voire une "politique actuelle ${ }^{47}$. Mais cette idéologie risque de devenir " caduque ", traditionnelle et déphasée ; même la philosophie de la praxis ${ }^{48}$ n'échappera pas à cette " caducité de tout système idéologique corrélative de l'affirmation de la validité historique de tout système, et de sa nécessité propre.... ${ }^{49}$. En quoi une philosophie est-elle organique ou nécessaire? En ce qu'elle est la " catharsis d'une vie pratique donnée " ${ }^{50}$, elle est reflet et compréhension des contradictions de la structure et ce contrairement

44. Q. $13,[1]$, p. 1556, C. p. 354.

45. Q. 10, II, [28], p. 1266, C. p. 71.

46. Q. 13, [37], p. 1642, C. p. 438.

47. Q. 10, II, [41], [1], p. 1295, C. p. 97.

48. Q. $11,[62]$, p. 1489, C. p. 284.

49. Q. $13,[10]$, p. 1570 , C. p. 367.

50. Q. $10, \mathrm{I},[10]$, p. 1231 , C. p. 37. 
aux autres idéologies (traditionnelles) qui sont des « créations inorganiques parce que contradictoires, parce que visant à concilier des intérêts opposés et contradictoires ... " ${ }^{51}$.

Qu'en est-il du travail du philosophe ? Ce travail a pour point de départ et point d'arrivée le sens commun ${ }^{52}$; il s'agit de travailler sur le sens commun afin de le rendre plus cohérent et plus critique :

. . . il s'agit par conséquent d'élaborer une philosophie qui ayant déjà une certaine diffusion, ou diffusivité, du fait qu'elle est liée à la vie pratique et implicite en elle, devienne un sens commun rénové avec la cohérence et le nerf des philosophies individuelles : cela ne peut arriver si n'est pas constamment perçue l'exigence du contact avec les " simples ". ${ }^{53}$

Par ce travail pédagogique, le sens commun gagne une « valeur intellectuelle » et la philosophie acquiert une valeur historique.

Nous devons dès lors envisager le travail philosophique en termes d'hégémonie philosophique, plus précisément dans le programme gramscien d'une " révolution culturelle et morale "; le philosophe s'y sent perdu sans ses racines dans l'histoire de la philosophie mais il y trouve quelqu'un à entendre, quelqu'un à qui parler et enfin la philosophie y gagnera-t-elle de la profondeur, car, comme l'écrivait Jacques Beaudry :

Tout se passe comme si on procédait, en philosophie, à un " détournement de fond » au profit de la surface. Tout est extérieur. Nous dissimulons notre fuite du risque de penser l'ici, notre ignorance, notre refus d'assumer ce réel mêlé et difficile ou notre incapacité à saisir ce qu'il y a de plus près de nous, dans le refuge des concepts et sous le fard des catégories importées. $^{54}$

Département de philosophie

Collège de Sherbrooke et Université de Sherbrooke

51. Q. 10, II, [41], note XII, pp. 1319-1320, C. p. 119.

52. Voir: G. Nardone, op. cit., p. 205.

53. Q. 11, [12], note III, pp. 1382-1383, C. p. 182.

54. Jacques Beaudry, "La philosophie comme chantier ou d'abord marche le bois, ensuite tu écriras ", Fragments, no 1 (octobre 1982), [p. 2]. 